\title{
Crystal growth, structural and optical characterization of a semi-organic single crystal for frequency conversion applications
}

\author{
P. Anandan ${ }^{\mathrm{a}, \mathrm{b}, *}, \mathrm{G}$. Parthipan ${ }^{\mathrm{b}}$, T. Saravanan ${ }^{\mathrm{b}}, \mathrm{R}$. Mohan Kumar $^{\mathrm{c}}$, G. Bhagavannarayana $^{\mathrm{d}}$, R. Jayavel ${ }^{\mathrm{a}}$ \\ ${ }^{a}$ Crystal Growth Centre, Anna University, Chennai 600 025, India \\ ${ }^{\mathrm{b}}$ Department of Physics, Thiruvalluvar College of Engineering and Technology, Vandhavasi 604 505, India \\ ' Department of Physics, Presidency College, Chennai 600 005, India \\ ${ }^{\mathrm{d}}$ Materials Characterization Division, National Physical Laboratory, Dr. K.S. Krishnan Road, New Delhi 110 012, India
}

\begin{abstract}
A B S T R A C T
Single crystals of semi-organic L-histidine hydrobromide have been grown by slow evaporation technique from a mixture of L-histidine and hydrobromic acid in aqueous solution at ambient temperature. From high-resolution X-ray diffraction analysis, the crystalline perfection of the grown crystal has been studied. Single crystal X-ray diffraction analyses, Nuclear Magnetic Resonance spectral analysis, Thermo-Gravimetry (TG), Differential Thermal Analysis (DTA) and hardness test have been employed to characterize the as-grown crystals. The UV cutoff wavelength of the grown crystal is below $300 \mathrm{~nm}$ and has a wide transparency window, which is suitable for second harmonic generation of lase in the blue region. Nonlinear optical characteristics have been studied using Q switched Nd:YAG lase $(\lambda=1064 \mathrm{~nm})$. The second harmonic generation conversion efficiency of the grown crystals confirms their suitability for frequency conversion applications.
\end{abstract}

\section{Introduction}

In recent decades, growth of nonlinear optical crystals has been given much importance, because of their potential applications like wave guides, frequency conversion devices, parametric oscillators, etc. Discovery of semi-organic nonlinear properties in L-arginine phosphate (LAP) has played a major role in both attracting the attention to crystalline salts of amino acids and working out the concept of semi-organic crystals [1]. Petrosyan et al. [2] derived some semi-organic nonlinear optical crystals of L-histidine salts and reported the interaction with various acids. Recently our group has reported the effect of solvent normality on the growth of L-tyrosine bromide single crystals [3]. Mukerji and Kar [4] have grown and investigated the structural, thermal and spectroscopic properties of L-arginine hydrobromide single crystals. Only a few papers have reported the growth and properties of L-histidine hydrobromide (LHHB) single crystals [5-7]. Ben Ahmed et al. [8] studied the structural and theoretical studies of the same.

In the present work we succeeded in growing L-histidine hydrobromide (LHHB) single crystals of comparatively larger size than the previous ones by slow evaporation solution growth technique (SEST). Furthermore, the grown LHHB single crystal has been observed to have large second harmonic generation conversion efficiency and it is better than that of KDP. The crystalline perfection of the grown crystal was studied by highresolution X-ray diffraction (HRXRD) analysis.

\section{Experimental}

\subsection{Synthesis and growth of L-histidine hydrobromide (LHHB)}

The analytical grade L-histidine (99\% pure) and Merck product of hydrobromic acid $(\mathrm{HBr})$ were taken as starting materials to synthesize L-histidine hydrobromide (LHHB). The calculated amount of hydrobromic acid was dissolved in deionized water of resistivity $18.2 \mathrm{M} \Omega \mathrm{cm}$. The measured amount of L-histidine was added to the solution slowly with stirring. The prepared solution was kept in a constant temperature bath controlled to an accuracy of $\pm 0.01{ }^{\circ} \mathrm{C}$ and allowed to evaporate at room temperature. The seed obtained by slow evaporation method was used for the bulk growth by slow evaporation technique. The microbes were not observed during the growth of LHHB, probably due to the presence of hydrobromic acid ( $\mathrm{HBr})$, which acts destructively on the growth of microbes. Bulk crystals of dimension $25 \times 20 \times 11 \mathrm{~mm}^{3}$ were harvested after a typical 
growth period of 30 days. The grown crystal was stable at ambient temperature and was non-hygroscopic. The as-grown crystal is shown in Fig. 1.

\subsection{Characterization techniques}

The crystalline perfection of the grown single crystal was studied by HRXRD by employing a multi-crystal X-ray diffractometer developed at NPL [9]. The well-collimated and monochromated $\mathrm{MoK}_{\alpha 1}$ beam obtained from the three monochromator Si crystals set in dispersive $(+,-,-)$ configuration was used as the exploring X-ray beam. The specimen crystal was aligned in the $(+,-,-,+)$ configuration. Due to the dispersive configuration, though lattice constants of the monochromator crystal(s) and the specimen are different, the unwanted dispersion broadening in the diffraction curve (DC) of the specimen crystal is insignificant. The specimen can be rotated about the vertical axis, which is perpendicular to the plane of diffraction, with minimum angular interval of 0.4 arcsec. Rocking or diffraction curves were recorded by changing the glancing angle (angle between the incident X-ray beam and the surface of the specimen) around the Bragg diffraction peak position $\theta_{\mathrm{B}}$ (taken as zero for the sake of convenience) starting from a suitable arbitrary glancing angle and ending at a glancing angle after the peak so that all the meaningful scattered intensities on both sides of the peak can be included in the diffraction curve. The DC was recorded by the so-called $\omega$ scan, wherein the detector was kept at the same angular position $2 \theta_{\mathrm{B}}$ with a wide opening for its slit. This arrangement is very appropriate to record the short range order scattering caused by the defects or the scattering from local Bragg diffractions by agglomerated point defects or low and very low angle structural grain boundaries [10]. Before recording the diffraction curve, the specimen was first lapped and chemically etched in a non-preferential etchant of water and acetone mixture in 1:2 volume ratio to remove the non-crystallized solute atoms remaining on the surface of the crystal and the possible layers, which may sometimes form on the surfaces of crystals grown by solution methods [11], and also to ensure the surface planarity.

Single crystal X-ray diffraction analysis was carried out using an ENRAF CAD-4 diffractometer with $\operatorname{MoK}_{\alpha}(\lambda=0.7107 \AA)$ radiation to identify the crystal system and to estimate the lattice parameter values. The FTIR spectrum of LHHB crystal was recorded in the range $400-4000 \mathrm{~cm}^{-1}$ using a Perkin-Elmer spectrometer by $\mathrm{KBr}$ pellet method in order to study the presence of various functional groups. The FT-Raman spectrum was recorded using a Bruker IFS66V FT-Raman spectrometer. The ${ }^{13} \mathrm{C}$

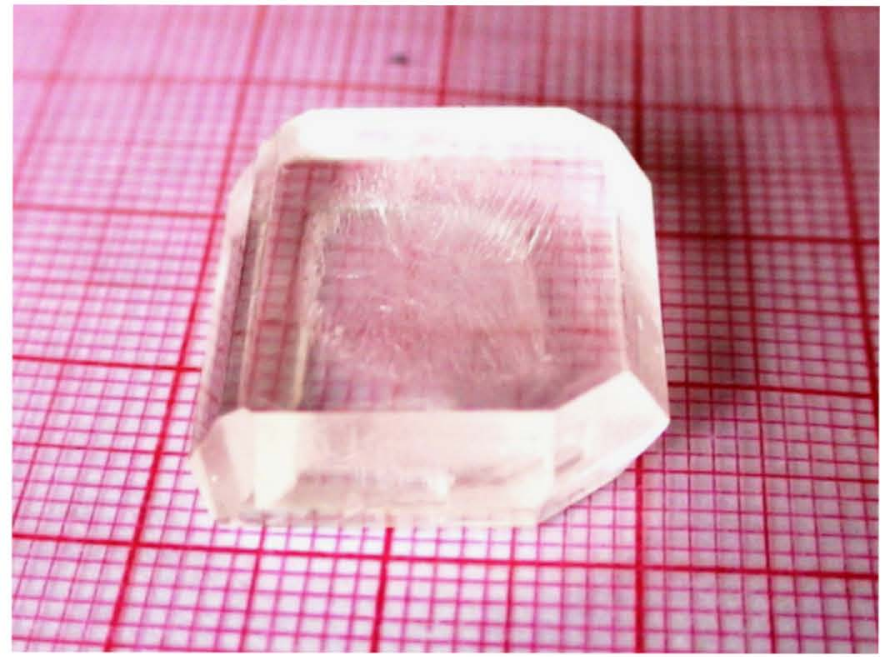

Fig. 1. Photograph of as-grown crystal of LHHB.
NMR spectrum of LHHB crystal was recorded using a JEOL GSX 400 instrument at $30^{\circ} \mathrm{C}$. Linear optical properties of the crystals were studied using a UV-visible spectrophotometer. To confirm the nonlinear optical property, the grown crystals were subjected to Kurtz powder SHG test [12]. Hardness of the grown crystal was studied by Vickers's micro-hardness test. The refractive index of the grown crystal was measured using a Metricon 2010 prism coupler instrument. Thermo-Gravimetry (TG), Derivative ThermoGravimetric (DTG) and Differential Thermal Analysis (DTA) for LHHB crystals were carried out in nitrogen atmosphere by a NETZSCH Thermal Analyzer to study the thermal properties of the as-grown crystal.

\section{Results and discussion}

\subsection{High-resolution $X$-ray diffraction study}

Fig. 2 shows the high-resolution diffraction curve (DC) recorded for a typical SEST grown LHHB single crystal using (0 23 3) diffracting planes in symmetrical Bragg geometry by employing the multi-crystal X-ray diffractometer with $\mathrm{MoK}_{\alpha 1}$ radiation. As seen in the figure, the DC contains a single peak, which indicates that the specimen is free from structural grain boundaries. The full width at half maximum (FWHM) of the curve is 18 arcsec, which is only slightly more than that expected for an ideally perfect crystal according to the plane wave theory of dynamical X-ray diffraction [13], but close to that expected for a nearly perfect real crystal.

It is important to note the asymmetry of the DC. For a particular angular deviation $(\Delta \theta)$ of glancing angle with respect to the peak position, the scattered intensity is much more in the positive direction as compared to that in the negative direction. This feature clearly indicates that the interstitial type of defects are predominant in the crystal than the vacancy defects. This can be well understood by the fact that due to interstitial defects (selfinterstitials or impurities at interstitial sites), which may be due to fast growth and/or impurities present in the raw material, the lattice around these defects undergoes compressive stress [14] and the lattice parameter $d$ (inter-planar spacing) decreases and leads to more scattered (also known as diffuse X-ray scattering) intensity at slightly higher Bragg angles $\left(\theta_{\mathrm{B}}\right)$ as $d$ and $\sin \theta_{\mathrm{B}}$ are inversely proportional to each other in the Bragg

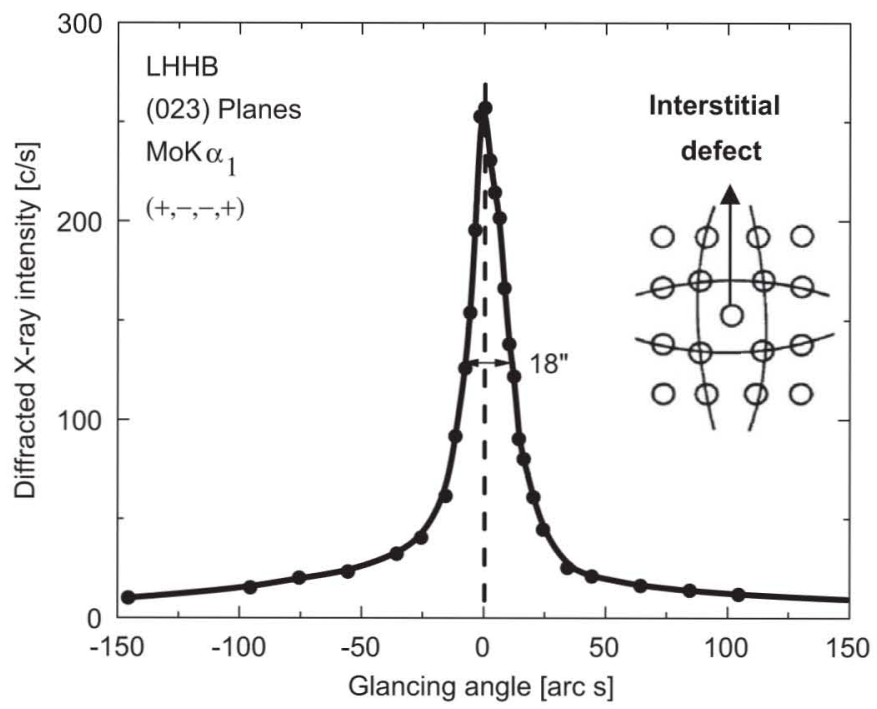

Fig. 2. High-resolution X-ray diffraction curve recorded for a typical LHHB single crystal specimen using $\left(\begin{array}{lll}0 & 2 & 3\end{array}\right)$ diffracting planes. 
equation ( $2 d \sin \theta_{\mathrm{B}}=n \lambda, n$ and $\lambda$ being the order of reflection and wavelength, respectively, which are fixed). However, the single diffraction curve with reasonably low FWHM indicates that the

Table 1

Crystal data of LHHB.

\begin{tabular}{ll}
\hline Identification code & Single crystal data \\
\hline Empirical formula & $\mathrm{C}_{6} \mathrm{H}_{10} \mathrm{~N}_{3} \mathrm{O}_{2} \mathrm{Br}$ \\
Temperature & $293 \mathrm{~K}$ \\
Wavelength & $0.7107 \AA$ \\
Crystal system & Orthorhombic \\
Space group & $\mathrm{P} 2{ }_{1} 2_{1} 2_{1}$ \\
Unit cell dimension & $a=7.046 \AA$ \\
& $b=9.061 \AA$ \\
& $c=15.271 \AA$ \\
Volume $\left(\AA^{3}\right)$ & 974.959 \\
Density, $\boldsymbol{\rho}\left(\mathbf{g} / \mathbf{c m}^{\mathbf{3}}\right)$ & 1.73 \\
\hline
\end{tabular}

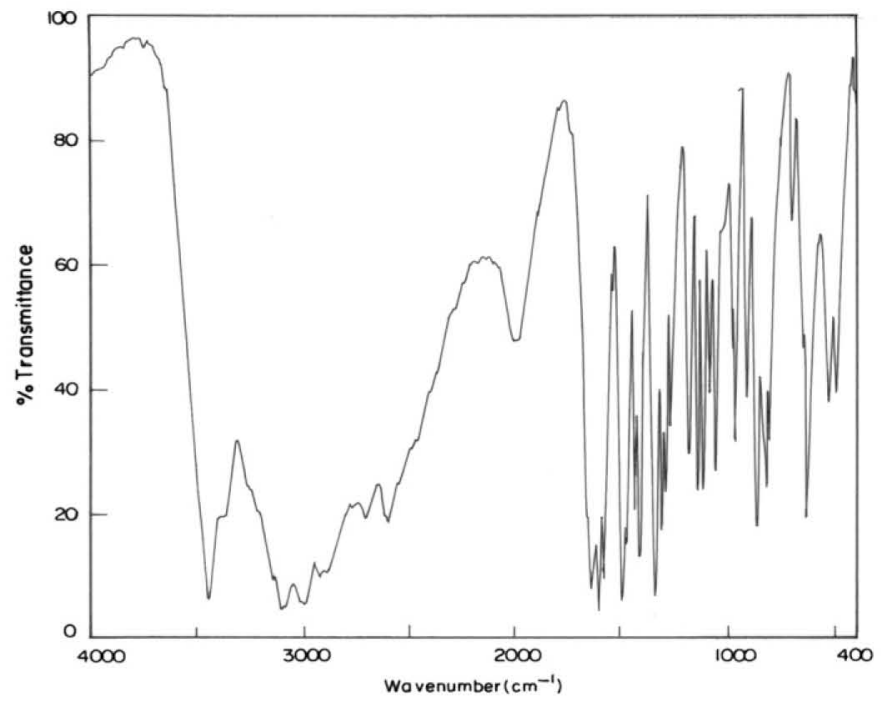

Fig. 3. FTIR spectrum of LHHB. crystalline perfection is excellent. The density of such interstitial defects is however very low and in almost all real crystals, including nature gifted crystals, these defects are commonly observed and are many times unavoidable due to thermodynamic conditions and hardly affect the device performance. More details may be obtained from the study of high-resolution diffuse X-ray scattering measurements [9], which are however not the main focus of the present investigation. It is worth mentioning here that the observed scattering due to interstitial defects is of short order nature as the strain due to such minute defects is limited to the very defective core, long order could not be expected and hence the change in the lattice parameter of the crystal is not possible. It may be mentioned here that the minute information like the asymmetry in the DC is possible in the present sample only because of the high resolution of the multi-crystal X-ray diffractometer used in the present investigation.

\subsection{Single crystal X-ray diffraction study}

Single crystal X-ray diffraction analysis has been carried out to determine the lattice parameters. From the single crystal XRD analysis, it was observed that the grown crystals possess orthorhombic structure with $\mathrm{P} 2{ }_{1} 2_{1} 2_{1}$ space group and the lattice parameter values are $a=7.046 \AA, b=9.061 \AA$ and $c=15.271 \AA$. The single crystal data are in good agreement with reported values [5], and are shown in Table 1.

\subsection{Fourier transform infrared and FT-Raman studies}

In order to analyze the presence of various functional groups in LHHB qualitatively, Fourier transform infrared (FTIR) and FT-Raman spectra have been recorded and are shown in Figs. 3 and 4, respectively. The absorption peaks from 2000 to $3500 \mathrm{~cm}^{-1}$ include overlapping of absorption peaks due to $\mathrm{O}-\mathrm{H}$ stretch of $-\mathrm{COOH}$ and $\mathrm{N}-\mathrm{H}$ stretch of $\mathrm{NH}_{3}^{+}$. The $\mathrm{CH}_{2}$ vibrations, which generally lie just below $3000 \mathrm{~cm}^{-1}$, are not clearly resolved in the FTIR spectrum. The broadening of absorption peak is due to hydrogen bonding. These absorption peaks are clearly resolved in the FT-Raman spectrum. The $\mathrm{CH}_{2}$ bending mode appears at

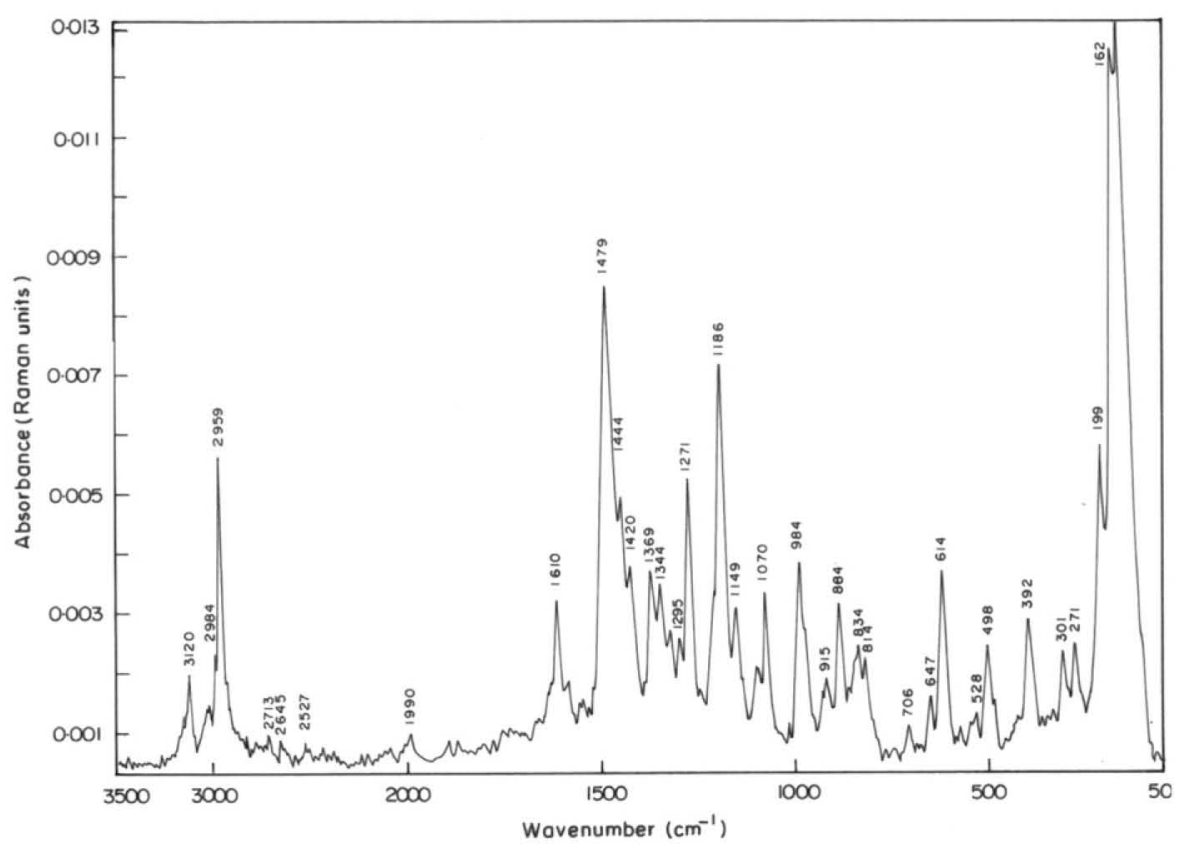

Fig. 4. FT-Raman spectrum of LHHB. 
$1335 \mathrm{~cm}^{-1}$ in the FTIR spectrum and it is at $1344 \mathrm{~cm}^{-1}$ in the Raman spectrum. The $\mathrm{C}=\mathrm{O}$ vibration of $-\mathrm{COOH}$ lies at $1636 \mathrm{~cm}^{-1}$ in the FTIR spectrum, whereas in the Raman spectrum it lies at $1610 \mathrm{~cm}^{-1}$. The torsional oscillation of $\mathrm{NH}_{3}^{+}$occurs nearly at $527 \mathrm{~cm}^{-1}$ in both the spectra. The wave number and the assignments for FTIR and FT-Raman spectra are presented in Table 2.

\section{4. ${ }^{13} \mathrm{C}$ nuclear magnetic resonance spectral study}

NMR spectrum has been used to identify the carbon atom present in various chemical environments and to confirm the functional groups in the grown LHHB crystal. Fig. 5 shows the recorded ${ }^{13} \mathrm{C}$ NMR spectrum. The carboxyl carbon gives its signal at $172.5 \mathrm{ppm}$. The aliphatic carbon carrying $\mathrm{NH}_{3}^{+}$and imidazole ring gives its signal at 53.54 and $25.75 \mathrm{ppm}$, respectively. The imidazole ring carbon produces their characteristic signal between 110 and $140 \mathrm{ppm}$. The ring carbon carrying the alkyl position gives its signal at $127.39 \mathrm{ppm}$. The ortho- and metacarbon of the ring with respective alkyl substituent give their signal at 134 and $117.72 \mathrm{ppm}$, respectively. As no other peaks are observed, the compound has been successfully synthesized and it is LHHB as a pure material.

\subsection{Linear and nonlinear optical property study}

Since absorption, if any, in the NLO material near the fundamental or second harmonic leads to loss of conversion efficiency, it is necessary to have the crystal with good optical transparency in the UV region. Optical transmission spectrum has been recorded in the range between 300 and $1200 \mathrm{~nm}$ for the LHHB crystals. The crystals possess UV cutoff below $300 \mathrm{~nm}$ and there is no absorption in the visible range of the spectrum. Hence, LHHB is useful for optoelectronic applications and a second harmonic generation (frequency conversion) from the Nd:YAG

Table 2

Wave numbers $\left(\mathrm{cm}^{-1}\right)$ of FTIR absorption peaks and FT- Raman lines in the spectra of LHHB Crystal.

\begin{tabular}{|c|c|c|}
\hline \multicolumn{2}{|c|}{ Wave number $\left(\mathrm{cm}^{-1}\right)$} & \multirow[t]{2}{*}{ Assignment } \\
\hline FTIR & FT-Raman & \\
\hline 3443 & - & $\mathrm{N}-\mathrm{H}$ asym. stretching \\
\hline 3102 & 3150 & N-H sym. stretching \\
\hline 3000 & 3010 & $\mathrm{C}-\mathrm{H}$ stretching \\
\hline 2955 & 2984 & $\mathrm{CH}_{2}$ asym. stretching \\
\hline 2601 & - & $\mathrm{NH}_{2}^{+}$asym. and sym. stretching \\
\hline 1636 & 1610 & $\mathrm{C}=\mathrm{O}$ stretching \\
\hline 1574 & - & $\mathrm{NH}_{3}^{+}$stretching \\
\hline 1492 & 1479 & $\mathrm{NH}_{3}^{+}$bending \\
\hline 1431 & 1420 & C-N stretching \\
\hline 1412 & - & $\mathrm{N}-\mathrm{H}$ bending \\
\hline 1335 & 1344 & $\mathrm{CH}_{2}$ deformation \\
\hline 1304 & 1295 & $\mathrm{NH}_{3}^{+}$in plane bending \\
\hline 1160 & 1186 & $\mathrm{C}-\mathrm{H}$ deformation \\
\hline- & 1149 & $\mathrm{C}-\mathrm{H}$ in plane bending \\
\hline 959 & 984 & $\mathrm{~N}-\mathrm{H}$ deformation \\
\hline 914 & 915 & $\mathrm{C}-\mathrm{H}$ out of plane bending \\
\hline 865 & 884 & $\mathrm{C}-\mathrm{N}$ deformation \\
\hline 822 & 834 & Ring deformation \\
\hline 804 & 814 & $\mathrm{C}-\mathrm{N}$ deformation \\
\hline 696 & 706 & $\mathrm{C}=\mathrm{O}$ deformation \\
\hline 628 & 647,614 & Ring deformation or $\mathrm{C}-\mathrm{N}$ deformation \\
\hline 527 & 528 & Torsion oscillation of $\mathrm{NH}_{3}^{+}$ \\
\hline 490 & 498 & $\mathrm{O}-\mathrm{H}$ in plane bending \\
\hline - & 392 & $\mathrm{C}-\mathrm{C}$ twisting \\
\hline - & 271 & C-N deformation \\
\hline
\end{tabular}

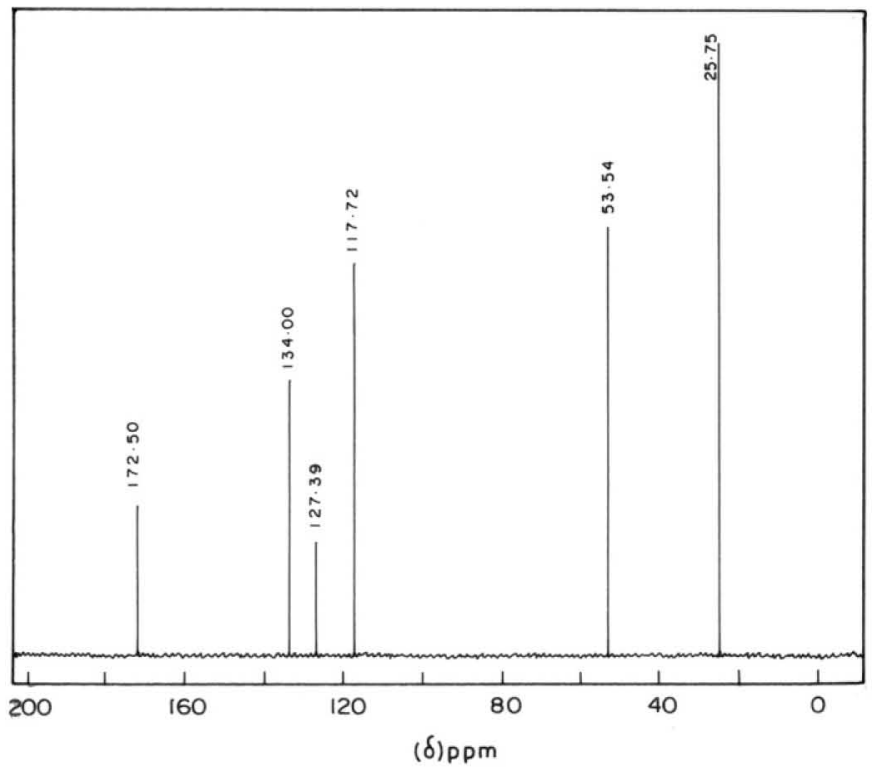

Fig. 5. ${ }^{13} \mathrm{C}$ NMR spectrum of LHHB.

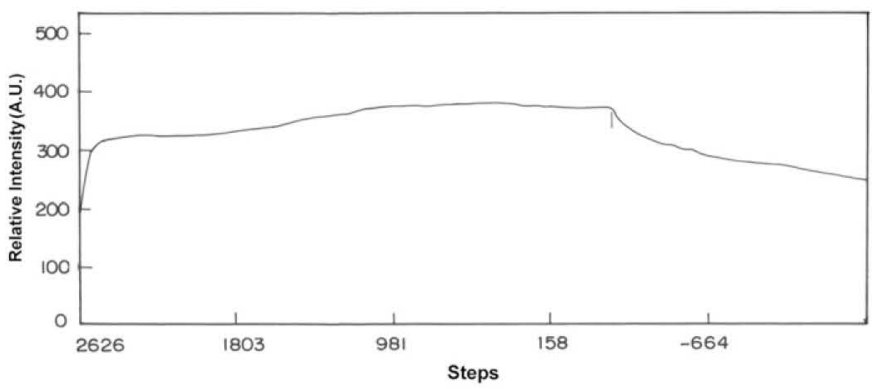

Fig. 6. Plot of angle of incidence vs. intensity.

Laser. The SHG efficiency of LHHB was studied by Kurtz and Perry's [12] powder technique. The powder sample prepared from the grown crystal has been subjected to the SHG test and the efficiency of the energy (frequency) conversion is confirmed by the emission of green light from the powder sample. A KDP sample was used as the reference material and output power intensity of LHHB has been found to be 1.6 times that of KDP.

\subsection{Refractive index study}

To measure the refractive index, the mirror polished crystal of size $10 \times 10 \times 2 \mathrm{~mm}^{3}$ was used. This sample was clamped against the prism and the index was determined by measuring the critical angle $\theta_{c}$ for the sample-prism interface. Nitrogen gas pressure was used to make a contact between the crystal and the prism. The bulk crystals usually show a clear critical angle knee, from which the refractive index have been calculated automatically. The knee observed is shown in Fig. 6 and the calculated refractive index is 1.6393 .

\subsection{Vickers' micro-hardness study}

Hardness of the grown crystal was estimated by Vickers' micro-hardness test. The indentations were made at room temperature on the prominent plane with a constant dwell time of $5 \mathrm{~s}$. The distance between two indentation points was 


\section{Acknowledgement}

One of the authors (P.A.) is grateful to Mrs. Balamani Arunachalam, Chairperson, Thiruvalluvar College of Engineering and Technology, Vandhavasi 604505, India, for her constant encouragement and support. The authors are thankful to Professor D. Rajan Babu, VIT University, Tamil Nadu, for his help in the measurement of refractive index.

\section{References}

[1] D. Xu, M. Jiang, Z. Tan, Acta Chem. Sin. 41 (1983) 570.

[2] H.A. Petrosyan, H.A. Karapetyan, M. Yu Antipin, A.M. Petrosyan, J. Cryst. Growth 275 (2005) e1919.

[3] P. Anandan, T. Saravanan, S. Vasudevan, R. Mohan Kumar, R. Jayavel, J. Cryst. Growth 312 (2010) 837.
[4] S. Mukerji, T. Kar, Mater. Res. Bull. 33 (1998) 619.

[5] Reena Ittyachan, P. Sagayaraj, J. Cryst. Growth 249 (2003) 557.

[6] K.V. Rajendran, D. Jayaraman, R. Jayavel, P. Ramasamy, J. Cryst. Growth 255 (2003) 361.

[7] N. Vijayan, G. Bhagavannarayana, K. Nagarajan, V. Upadhyaya, Mater. Chem. Phys. 115 (2009) 656.

[8] A. Ben Ahmed, H. Feki, Y. Abid, H. Boughzala, A. Mlayah, J. Mol. Struct. 888 (2008) 180.

[9] Lal Krishan, G. Bhagavannarayana, J. Appl. Crystallogr 22 (1989) 209.

[10] G. Bhagavannarayana, S.K. Kushwaha, J. Appl. Crystallogr. 43 (2010) 154.

[11] G. Bhagavannarayana, S. Parthiban, Subbiah Meenakshisundaram, J. Appl. Crystallogr. 39 (2006) 784.

[12] S.K. Kurtz, T.T. Perry, J. Appl. Phys. 39 (1968) 3798.

[13] B.W. Batterman, H. Cole, Rev. Mod. Phys. 36 (1964) 681.

[14] G. Bhagavannarayana, S. Parthiban, Subbiah Meenakshisundaram, Cryst. Growth Des. 8 (2008) 446.

[15] D. Kalaiselvi, R. Mohan Kumar, R. Jayavel, Mater. Lett. 62 (2008) 755.

[16] R. Rajasekaran, P.M. Ushasree, R. Jayavel, P. Ramasamy, J. Cryst. Growth 229 (2001) 563. 